\title{
A Cockcroft-Walton High-Voltage Power Supply for the EUSO Instruments
}

\section{S. Bacholle*, C. Blaksley, P. Gorodetzky}

Laboratoire Astroparticule et Cosmologie (APC), Université Paris 7/CNRS, 10 rue A. Domon et

L. Duquet, 75205 Paris Cedex 13, France

E-mail: philippe.gorodetzky@cern.ch

\section{J. Karczmarczyk, J. Szabelski}

NCBJ, Cosmic Ray Laboratory, PL-90-950, Lodz, Poland

\section{for the JEM-EUSO Collaboration}

The future JEM-EUSO instrument is a UV telescope to be installed on the International Space Station (ISS) with the goal of observing Extensive Air Showers created by Ultra-High Energy Cosmic rays. EUSO-balloon is a pathfinder mission for JEM-EUSO which flew in a stratospheric balloon from Timmins, Canada in August 2014. Due to its placement on the ISS, two major specifications of the JEM-EUSO instrument are that: i) the power allocated for the entire instrument should be no more than $1000 \mathrm{~W}$; requiring that the power allocated to polarize the Photomultiplier Tubes (PMTs) should be less than $\sim 50 \mathrm{~W}$ (using normal resistive voltage dividers requires nearly $2 \mathrm{~kW}$ ), and ii) the light intensity reaching JEM-EUSO has a dynamic range larger than $10^{6}$, going from the background illumination (about one photoelectron per pixel per $2.5 \mu \mathrm{s}$ ) up to Transient Luminous objects. Our solution for i) is to use a Cockcroft-Walton circuit to effectively create a separate power supply for each dynode, regrouping identical dynodes at the same power supply. These groups will be at the level of the Elementary Cell (EC, 4 PMTs). The required dynamic range will be provided by a switching circuit, giving an overall dynamic range of $10^{6}$ in steps of $10^{2}$ and in a time of several microseconds. Here we present the Cockcroft-Walton high voltage power supply design used in EUSO-Balloon. This design resulted in a power consumption of 630 $\mathrm{mW}$ to polarize the entire EUSO-Balloon focal surface (36 PMTs), and the switching circuit was capable of reducing the focal surface collection efficiency within $2.5 \mu \mathrm{s}$.

The 34th International Cosmic Ray Conference,

30 July- 6 August, 2015

The Hague, The Netherlands

\footnotetext{
* Speaker.
} 


\section{Introduction}

The Japanese Experiment Module-Extreme Universe Space Observatory (JEM-EUSO) telescope is intended to measure the highest energy cosmic rays (so called Ultra-High Energy Cosmic rays (UHECR)) by monitoring the Earth's atmosphere from the International Space Station (ISS) [1]. The advantage of this approach is the huge geometrical factor, as an area with a radius of about $200 \mathrm{~km}$ would be monitored. The JEM-EUSO instrument is to consist of a UV telescope (lenses and focal surface) with a nearly circular area of about 2.3 meters in diameter. The light detector must be very fast, in order to monitor the development of Extensive Air Showers (EAS), which typically last about $30 \mu \mathrm{s}$, and the angular resolution (pixelization) should be fine enough to see the spatial development of EAS from the 350-400 km altitude of ISS. In order to meet these requirements, the focal surface of the telescope will consist of 4932 Multi-Anode Photomultiplier Tubes (MAPMT), each with 64 anodes (i.e. 315648 pixels in total, each viewing $500 \mathrm{~m} \times 500$ $\mathrm{m}$ on ground), combined with a fast read-out based on the SPACIROC Application Specific Integrated Circuit [2]. For observation of UHECR, single photoelectrons (pe) will be counted in each pixel, and integrated every Gate Time Unit (GTU) of $2.5 \mu \mathrm{s}$. With variable atmosphere density, the JEM-EUSO instrument will work as a time projection chamber, allowing 3D reconstruction of EAS.

The EUSO-Balloon experiment [3] is a technical pathfinder for the JEM-EUSO mission. The EUSO-Balloon instrument is composed of a single Photodetection Module (PDM) with optics and a data-processing system [4] housed in a balloon-borne gondola. This instrument had a successful first flight to an altitude of $38 \mathrm{~km}$ in August 2014 from Timmins, Canada.

\subsection{Focal Surface Structure and Power Consumption}

The photodetector which will be used in JEM-EUSO is the Hamamatsu R11265-M64 MAPMT (M64). This MAPMT is a 12-stage vacuum PhotoMultiplier Tube (PMT) with an additional grid element near the anodes for better focusing of internal pe cascades. Four MAPMT form one Elementary Cell (EC) - the basic logical unit of the focal surface, and nine EC form one PDM $(6 \times 6$ MAPMT). The total focal surface of the JEM-EUSO instrument is expected to be composed of 137 PDMs.

One HVPS is planned for each EC. The M64 has a single photoelectron gain of $10^{6}$ for cathode bias voltages in the range of 900 to $1000 \mathrm{~V}$. As the telescope will view the night atmosphere with an ultra-violet filter, most of the measured light will come from the UV background in the telescope field of view. The average background rate is expected to be about 1 pe per pixel per GTU. The expected anode current due to the UV background will be approximately $4.1 \mu \mathrm{A}$ per MAPMT, and the anode current for one PDM will be $147 \mu \mathrm{A}$. This would give a background anode current of $20.2 \mathrm{~mA}$ for the entire JEM-EUSO focal surface.

The standard approach for powering a PMT is to use a resistive voltage divider in parallel with the PMT. The current in the divider should be more than 100 times the background anode current. The required divider current (or sum over all dividers) should then be $2.02 \mathrm{~A}$. As the voltage required for a gain of $10^{6}$ is at least $-900 \mathrm{~V}$, the power consumption of the voltage divider would be more than $1.8 \mathrm{~kW}$. This value is nearly twice the limit for JEM-EUSO power consumption for all instrument devices, and so the standard approach to powering PMTs is excluded. In addition, the 
power lost in a resistive voltage divider is dissipated as heat, which must then be removed from the focal surface.

\subsection{Photomultiplier Tube Gain and Dynamics}

As the standard approach to powering PMTs is excluded for JEM-EUSO, an alternative design philosophy is needed. In a PMT, the majority of the current is drawn by the later dynodes, which are at lower voltage. A large savings in power consumption can therefore be realized by supplying only the minimum current needed to the cathode; our solution is to use a Cockcroft-Walton voltage multiplier circuit [5]. High voltage power supplies based on the Cockcroft-Walton have previously been used to power PMTs (e.g. [6, 7, 8]), and the M64 is well suited to this type of power supply as the voltage repartition has an equal step between the majority of the dynodes.

There is also the question of the instruments dynamic range. Although the primary goal of JEM-EUSO is the observation of EAS, there are numerous other phenomena which can be studied by an Earth-viewing UV telescope. The dynamic range of the read-out electronics is limited, however, and the last dynode and the PMT anodes suffer radiation damage due to electron bombardment. This can affect the gain of the PMTs if the anode receives too high of an electron flux. The dynamic range between the other phenomena of interest and extensive air showers requires a factor of nearly $10^{6}$ difference in gain. In order to study the phenomena across this range of light levels, and to protect the PMTs from damage due to overexposure at high gain, there must be a system to lower the number of electrons reaching the anode within a few microseconds.

The single photoelectron gain of a PMT is determined by the repartition of voltages across its dynodes, and the magnitude of the gain varies logarithmically with the cathode bias voltage, $V_{\mathrm{c}}$, when the overall voltage repartition is scaled. Switching the gain in this way means that the voltage on 14 separate elements must be changed at once. This is slow due to the capacitance of the PMT, and is complicated due to the need to have 14 switch circuits in parallel (as we are not using a resistive voltage divider).

However, the current through the anode is proportional to the PMT gain, which is the product of the single photoelectron gain and the collection efficiency (c.f. [9] for further discussion), and the collection efficiency depends on the voltage difference between the photocathode and the first dynode. We can therefore reduce the current received by the anode of the PMT by changing only the voltage on the photocathode. In this case, the single photoelectron gain of the PMT will be roughly the same at each switch step, but the number of photoelectrons which will be collected into the multiplication stage of the PMT will be reduced. An advantage of this is that we can continue to use a single photon counting read-out with each step in gain offering a decrease in the collection efficiency. Furthermore, there is only one element to be switched, simplifying the design of the switching circuit.

\section{Tests of the CW-HVPS and Switch Prototype}

We tested a prototype of the CW-HVPS and the switches, before the final printed circuit boards (PCBs) were made for EUSO-Balloon. The CW-HVPS prototype board took an input voltage of $+28 \mathrm{~V}$, to power the PMTs, and $+3.3 \mathrm{~V}$ for the control logic and oscillator. An output regulation 


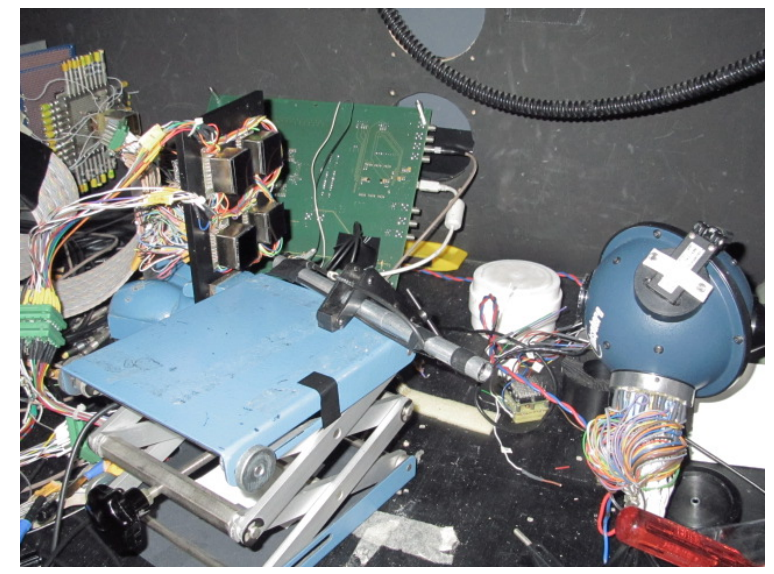

Figure 1: The test EC set up in our black box. The EC of 4 PMTs can be seen in the upper left of the picture, for this study of the power supply a simple prototype with large space between PMTs was sufficient. The background illumination was provided by the collection of LEDs which were attached to the integrating sphere. The 2nd collimated LED is in the center of the photograph, mounted on the blue collapsing stage with a clamp. This LED was aimed at the center of PMT-3 (the bottom left corner of the EC).

voltage between 0 and $2.44 \mathrm{~V}$ could be set on the board using a potentiometer, scaling the CWHVPS output voltage.

The switches are integrated directly into the CW-HVPS circuit. In the prototype board the switches were commanded by a second control board, allowing a change between four voltage levels: i) full cathode voltage, ii-iii) two different intermediate voltages, each taken from a resistive bridge between any two stages of the Cockcroft-Walton, and iv) the power supply ground.

These tests were the first to study the reduction in gain caused by changing only the cathode voltage of the M64 MAPMT, and were also the first in which we tested the CW-HVPS with a complete EC of four M64 PMTs. The test EC along with the rest of the test set-up are shown in Fig. 1. To control background light, we enclosed the entire set-up in a black box. We illuminated the EC using the output of an integrating sphere, and monitored the light level using a NIST-calibrated photodiode placed on one port of the sphere, as described in ref. [9].

Our Data Acquisition system (DAQ) was based on CERN prototypes of the C205 CAMAC QDC. These QDC modules measure 32 input charges of up to $900 \mathrm{pC}$ during a gate of between $100 \mathrm{~ns}$ and $5 \mu \mathrm{s}$ using a charge-to-voltage converter. The illumination on the EC was a combination of DC illumination over the entire EC using a collection of UV LEDs and an illumination on only a few pixels of one PMT using a second collimated LED. Using this setup, we measured the charge on PMT-3 each $2.5 \mu \mathrm{s}$ as a function of $I_{\mathrm{D} 12}$. The result of this measurement is shown in Fig. 2(b), and the current on dynode twelve was linear with the photoelectron rate.

During this measurement we also noted the current drawn by the +28 and +3.3 volt power supplies to the CW-HVPS board. The $+3.3 \mathrm{~V}$ is the supply for the control of the Cockcroft-Walton circuit and the $+28 \mathrm{~V}$ provides the power to the PMT. With the EC illuminated at the background level, $0.211 \mu \mathrm{W}$ on the NIST photodiode, we measured a current of $1.6 \mathrm{~mA}$ drawn from the +28 volt supply and a current of $7.6 \mathrm{~mA}$ from the 3.3 volt supply, giving a power consumption of $70 \mathrm{~mW}$ 

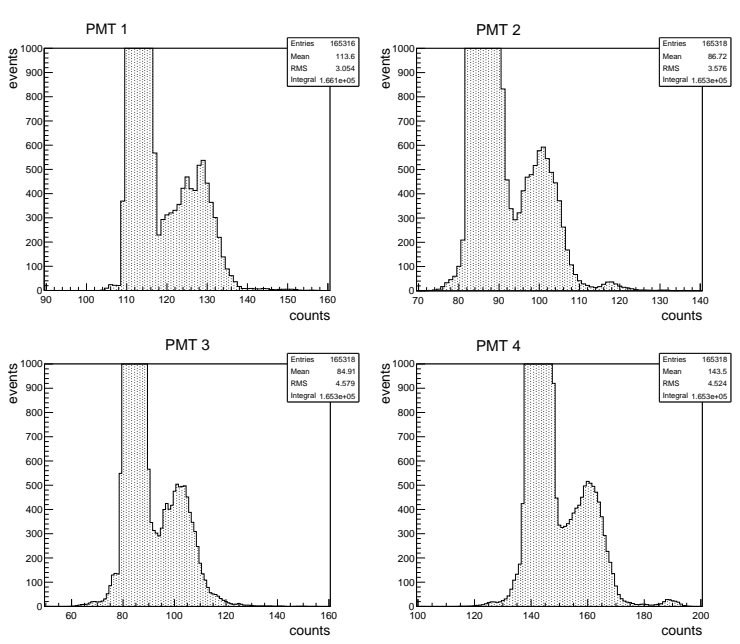

(a) Single Photoelectron Spectra of the EC with the CWHVPS

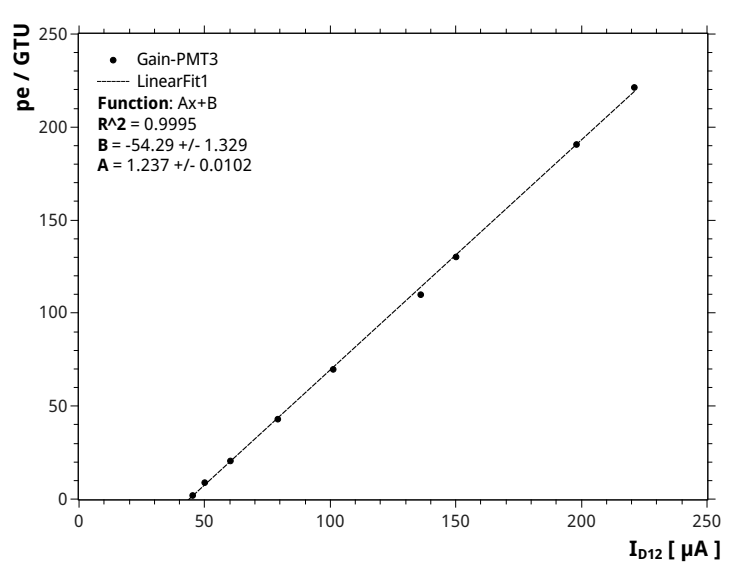

(b) Counting Rate Vs. Dynode Current

Figure 2: Fig. 2(a): The measured single photoelectron spectra for the four PMTs in the test EC. These spectra were taken with the EC powered by the CW-HVPS at a cathode voltage of $1000 \mathrm{~V}\left(V_{\text {reg }}=2.20\right)$.

Fig. 2(b): The counting rate versus the measured dynode current with the EC powered by the CW-HVPS. The count rate is given in pe per $2.5 \mu \mathrm{s}$ (GTU). The dashed line is a linear least-squares fit to the measured data. As can be seen from the fit, the linearity of the current with the counting rate is excellent from 1 pe/GTU up to and beyond 200 pe/GTU, showing the quality of the CW-HVPS.

per EC at twice the JEM-EUSO background illumination.

\section{Tests of the Switches and PMT Gain Reduction}

We also used the same set-up to test the switches. The control of the switches was by TTL levels and allowed us to apply one of four possible voltages to the photocathode: i) The full cathode voltage, depending on $V_{\text {reg }}$, but nominally $900 \mathrm{~V}\left(\right.$ Gain $\left.\simeq 110^{6}\right)$, ii) A voltage from a resistive bridge around $\mathrm{D} 1,739 \mathrm{~V}$ in this case (Gain $\simeq 110^{4}$ ), iii) A voltage from a resistive bridge between D8 and D9, here $250 \mathrm{~V}$ (Gain $\simeq 110^{2}$ ), and iv) $0 \mathrm{~V}$ (Gain $\simeq 10$ ). For this test, the TTL signal was a square pulse such that the switch on the $900 \mathrm{~V}$ supply went from "on" to "off" at intervals of 1.2 seconds, while the switch on the $739 \mathrm{~V}$ went from "off" to "on" at the same moment.

We checked the timing and function of the switches by looking at the anode signal of pixel 21 of PMT-3 on an oscilloscope. In our first test we switched the cathode voltage down from 900 $\mathrm{V}$ to $739 \mathrm{~V}$. The oscilloscope screen is shown in Fig. 3. The green line is the TTL command to the switches, and the golden-brown line is the anode signal from PMT-3. The switch functioned properly and the reduction in cathode voltage also reduces the current on the anode, as can be seen in Fig. 3(a). The same oscilloscope view with a time division of $2.0 \mu$ s is shown in Fig. 3(b), and the reduction in PMT gain occurred in less than $4 \mu \mathrm{s}$.

To test switching back up, we synchronized the second LED with the switch command, so that the LED was on during the switch down to 739 and powered off before the switch back up to 900 V. This is a better approximation of what would occur in JEM-EUSO, where the cathode voltage 


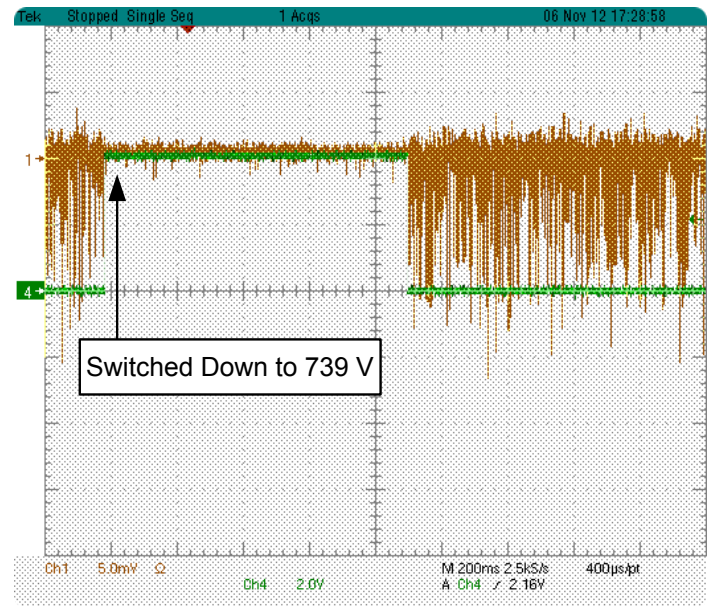

(a) $900 \mathrm{~V}$ to $739 \mathrm{~V}$ (200 ms/division)

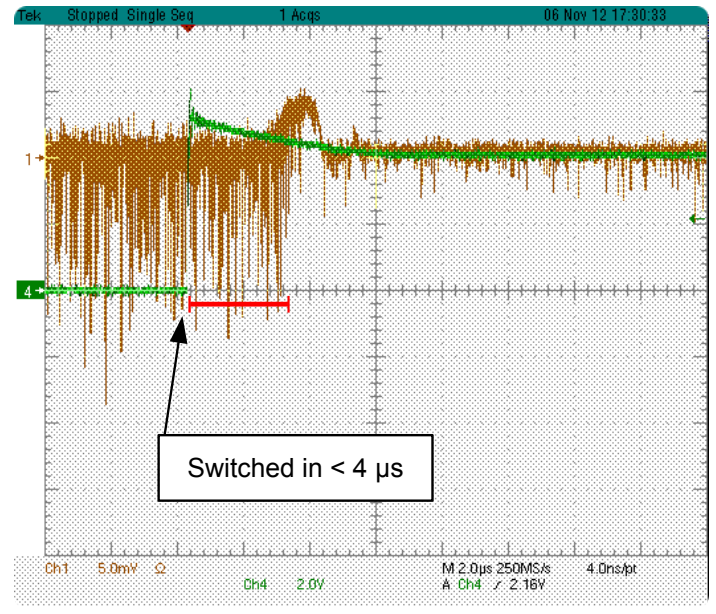

(b) $900 \mathrm{~V}$ to $739 \mathrm{~V}(2.0 \mu$ s/division $)$

Figure 3: Oscilloscope screen captures of the switch operation. The switch command is the green line. The switch period is 1.2 seconds, and the high TTL level sets the cathode voltage down to $739 \mathrm{~V}$ from 900 V. The anode signal of pixel 21 of PMT-3 of the test EC is shown by the brown line. The reduction in PMT gain can be clearly seen. As can be seen in Fig. 3(b) the gain is lowered in less than $4 \mu \mathrm{s}$.

would be switched down in response to a large signal in a few pixels, and then switched up as the signal settles back to the background level. In this case the gain recovered in less than $4 \mu \mathrm{s}$.

\subsection{PMT Gain Reduction}

After testing the basic function of the integrated switches, we studied the reduction in PMT gain caused by lowering the cathode voltage. For the first measurement, we powered the test EC using the CAEN HVPS with the voltage distribution of the CW-HVPS at $900 \mathrm{~V}$. Using the CAEN HVPS allowed us to set the cathode at an arbitrary voltage. We first measured the pedestals of all four PMT in the EC, and then illuminated the EC at the background level using the collection of LEDs with a few pixels of PMT-3 further illuminated using a second LED.

We then measured the charge received on the anode of pixel 21 of PMT-3 during a gate of $2.5 \mu \mathrm{s}$ (1 GTU), minus the pedestal charge, as a function of the cathode voltage. The charge on the anode is proportional to the PMT gain, i.e. the product of the single photoelectron gain and the collection efficiency. We increased the illumination several times as the cathode voltage was reduced, so that the anode signal could still be seen in the QDC as the PMT gain decreased. Each time we changed the light level, we remeasured the response at the same voltage in order to account for the change in the number of photons incident on the pixel per GTU.

We first studied the response for a cathode voltage between $900 \mathrm{~V}$ and $0 \mathrm{~V}$ (cathode grounded), shown in Fig. 4. Below $200 \mathrm{~V}$ the CAEN HVPS had difficulty polarizing properly, and so we did not make any measurements between $200 \mathrm{~V}$ and $0 \mathrm{~V}$. The dynamic range shown by the plot gives a factor of $5.210^{3}$ between 900 and $0 \mathrm{~V}$, and the dynamic range at a given gain is factor of $\sim 200$, as shown by Fig. 2(b). This gives a total dynamic range of more than $110^{6}$. 


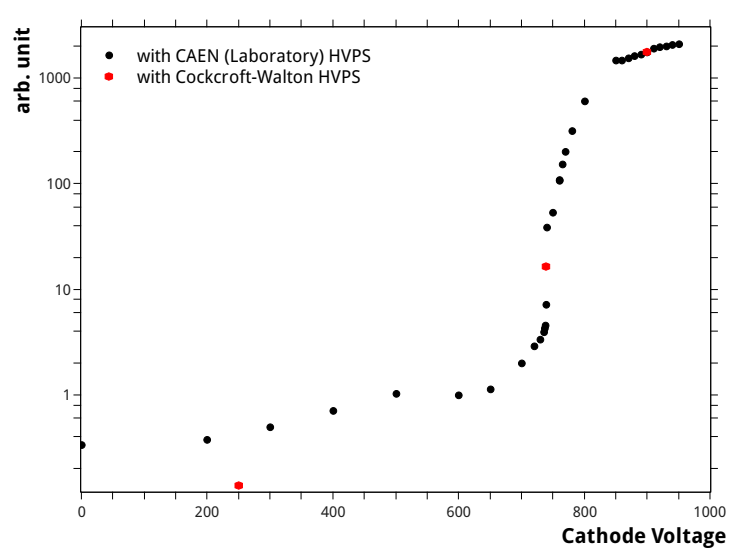

Figure 4: A plot of the reduction in PMT gain caused by changing only the cathode voltage. The cathode voltage is shown in abscissa, and the number of QDC counts minus the pedestal is shown in ordinates. The response in the QDC is proportional to the product of the single photoelectron gain and the number of photoelectrons, and thus to the PMT gain at a constant illumination. The full curve is taken using the CAEN HVPS, while the hexagonal markers show points measured using the CW-HVPS. The difference between the two measurements is likely due to the high impedance of the CAEN-HVPS.

We then repeated the same measurement using the CW-HVPS itself, this time at a cathode voltage given by the four switch levels: $900 \mathrm{~V}, 739 \mathrm{~V}, 250 \mathrm{~V}$ and $0 \mathrm{~V}$. These measurements are shown in Fig. 4 by the hexagonal markers. We corrected for the difference in illumination by normalizing the measured charge at $900 \mathrm{~V}$ to the previous value measured using the CAEN HVPS. This correction was less than 2\%. The reduction in PMT gain when using the CW-HVPS was similar to that seen when using the CAEN HVPS, but not exactly the same, with the difference being greater at lower voltages. This was most likely due to the trouble which the CAEN HVPS has polarizing at low voltages. With the CW-HVPS, we measured the gain reduction to be factor of 107 between $900 \mathrm{~V}$ and $739 \mathrm{~V}$, a factor of 118 between $739 \mathrm{~V}$ and $250 \mathrm{~V}$, and a further factor of 1.25 between $250 \mathrm{~V}$ and $0 \mathrm{~V}$. This gives a range of $1.610^{4}$, for a total dynamic range of $\simeq 310^{6}$.

\section{Conclusions}

Our conclusion from these tests on the Cockcroft-Walton HVPS prototype was that it not only worked as intended, but better. The single photoelectron gain of the M64 multianode PMT (MAPMT) using the CW-HVPS was greater than or equal to that with the normal Hamamatsu voltage repartition. The CW-HVPS was found to be quiet, and did not induce any unwanted noise on the anode signals of the test EC. In addition, the power supply does not saturate at 100 times the background illumination and shows a good linearity.

The primary concern of power consumption is also met: our test EC used $70 \mathrm{~mW}$ at three times the background illumination. As each EC will have one HVPS, this gives a power consumption of $630 \mathrm{~mW}$ for an entire photodetection module of $9 \mathrm{EC}$. This can be compared to $13 \mathrm{~W}$ for a resistive voltage divider. 
The switching circuit of the CW-HVPS was also found to work well, and it is capable of switching the cathode voltage down in 1 GTU (i.e. within $2.5 \mu$ s). Recovering back up to a higher cathode voltage is equally fast when not switching into a load. The four switch levels give an overall dynamics of i)1-200 photoelectrons per GTU at $900 \mathrm{~V}$, ii) 200 to $210^{4}$ photoelectrons per GTU at $739 \mathrm{~V}$, iii) $210^{4}$ to $210^{6}$ photoelectrons per GTU at $250 \mathrm{~V}$, and iv) $210^{6}$ to $\sim 310^{6}$ photoelectrons per GTU at $0 \mathrm{~V}$. This dynamic range allows the EUSO PDM to explore the full range of UV phenomena, including extensive air showers, meteoroids and meteors, lightning or other atmospheric emission, and human-made light.

Acknowledgment: We wish to thank the CNES and IN2P3 for their support of the EUSO Balloon project, within which this work was undertaken. Both the French and Polish authors would like to thank the support of the COPININ2P3 program. C. Blaksley would like to thank the RIKEN EUSO team for hosting him as a visiting researcher.

This work was partially supported by Basic Science Interdisciplinary Research Projects of RIKEN and JSPS KAKENHI Grant (22340063, 23340081, and 24244042), by the Italian Ministry of Foreign Affairs, General Direction for the Cultural Promotion and Cooperation, by the 'Helmholtz Alliance for Astroparticle Physics HAP' funded by the Initiative and Networking Fund of the Helmholtz Association, Germany, and by Slovak Academy of Sciences MVTS JEM-EUSO as well as VEGA grant agency project 2/0076/13. Russia is supported by the Russian Foundation for Basic Research Grant No 13-02-12175-ofi-m. The Spanish Consortium involved in the JEM-EUSO Space Mission is funded by MICINN \& MINECO under the Space Program projects: AYA2009-06037-E/AYA, AYA-ESP2010-19082, AYA-ESP2011-29489C03, AYA-ESP2012-39115-C03, AYA-ESP2013-47816-C4, MINECO/FEDER-UNAH13-4E-2741, CSD2009-00064 (Consolider MULTIDARK) and by Comunidad de Madrid (CAM) under projects S2009/ESP-1496 \& S2013/ICE-2822.

\section{References}

[1] J.H. Adams Jr., et al. An evaluation of the exposure in nadir observation of the jem-euso mission. Astroparticle Physics, 44(0):76 - 90, 2013.

[2] S. Ahmad, et al. SPACIROC: A rad-hard front-end readout chip for the JEM-EUSO telescope. JINST, 5:C12012, 2010.

[3] Giuseppe Osteria and Valentina Scotti. Euso-Balloon: A pathfinder mission for the JEM-EUSO experiment. Nucl.Instrum.Meth., A732:320-324, 2013.

[4] Valentina Scotti and G. Osteria. The data processor of the EUSO-Balloon experiment. JINST, 9:C03034, 2014.

[5] J. D. Cockcroft and E. T. S. Walton. Experiments with high velocity positive ions. ii. the disintegration of elements by high velocity protons. Proceedings of the Royal Society of London. Series A, 137(831):229-242, 1932.

[6] Bin Lu, Luke W. Mo, and Nunamaker Thomas A. The Cockcroft-Walton Photomultiplier Tube Base And The Ethernet High Voltage Controller. Technical Report VPI-IHEP-91/1, Institute of High Energy Physics, Physics Department Virginia Polytechnic Institute and State University, 1991.

[7] A. Brunner, et al. A Cockcroft-Walton Base for the FEU84-3 Photomultiplier Tube. Nucl.Instrum.Meth., A414:466-476, 1998.

[8] P. Timmer, E. Heine, and Peek H. Very low power, high voltage base for a Photo Multiplier Tube for the KM3NeT deep sea neutrino telescope. JINST, 5:C12049, 2010.

[9] C. Blaksley and P. Gorodetzky. A setup for the precision measurement of multianode photomultiplier efficiency. Nuclear Instruments and Methods in Physics Research Section A: Accelerators, Spectrometers, Detectors and Associated Equipment, 764(0):198 - 205, 2014. 